\title{
Two-Dimensional Analytical Solution of the Laminar Forced Convection in a Circular Duct with Periodic Boundary Condition
}

\author{
M. R. Astaraki and N. Ghiasi Tabari \\ Mechanical Engineering Department, Islamic Azad University, Dashtestan Branch, Dashtestan 7561888711, Iran \\ Correspondence should be addressed to N. Ghiasi Tabari, ghiasi@diau.ac.ir
}

Received 7 August 2012; Revised 6 November 2012; Accepted 7 November 2012

Academic Editor: Ahmet Z. Sahin

Copyright ( $) 2012$ M. R. Astaraki and N. Ghiasi Tabari. This is an open access article distributed under the Creative Commons Attribution License, which permits unrestricted use, distribution, and reproduction in any medium, provided the original work is properly cited.

In the present study analytical solution for forced convection heat transfer in a circular duct with a special boundary condition has been presented, because the external wall temperature is a periodic function of axial direction. Local energy balance equation is written with reference to the fully developed regime. Also governing equations are two-dimensionally solved, and the effect of duct wall thickness has been considered. The temperature distribution of fluid and solid phases is assumed as a periodic function of axial direction and finally temperature distribution in the flow field, solid wall, and local Nusselt number, is obtained analytically.

\section{Introduction}

In some convection and conduction heat transfer application, the temperature of boundaries is not constant and is a function of a dimension (X, Y, Z) or time. Some of these functions can be modeled as a periodic function. The mentioned boundary condition is applied in several engineering problems such as the nuclear reactor cooling system design and Stirling-cycle machines heat exchanger. Also in many industrial boilers which the heating up process is done by several burners, the above boundary condition is useful for numerical or analytical simulation.

Barletta et al. [1, 2] studied forced convection flow, respectively, with sinusoidal wall heat flux distribution and sinusoidal temperature distribution on the longitudinal boundary in a plane channel. Mentioned studies revealed that temperature distribution in longitudinal direction can be expressed as aperiodic function of the longitudinal coordinate. Also in [3] the effect of viscous dissipation on temperature in a circular duct in the case of a sinusoidal axial variation of the wall heat flux is evaluated.

In $[4,5]$ the laminar forced convection with the axial periodic boundary condition is studied by taking into account the axial heat conduction. Barletta et al. [6] investigated the effect of conducting wall in a parallel-plane channel. Their study showed that the increase in wall heat conductivity causes the increase in temperature oscillation amplitude off low field.

In addition to the analytical studies, some numerical studies are done on convection heat transfer with periodic boundary condition [7-11]. Periodic forced convection in the thermal entrance region of a porous medium is investigated in [12] by using finite element method. In mentioned that study, a two-temperature model employed in order to evaluate the solid and fluid phase temperatures.

In the present paper, the forced convection in a circular duct with the boundary condition given by a temperature distribution which varies axially with sinusoidal law is solved analytically. It should be mentioned the modeling is done by taking into account the heat conduction in the duct wall. The longitudinal heat conduction and the viscous dissipation in the fluid will be neglected. Reference will be made to the hydrodynamically and thermally developed region. The two-dimensional energy balance equations will be solved analytically both for the fluid and the solid region.

The fluid and solid temperature distribution will be obtained analytically by expressing the energy balance equation as a complex-valued hypergeometric confluent equation.

For the analytical solution, [2] is used as the main source. 


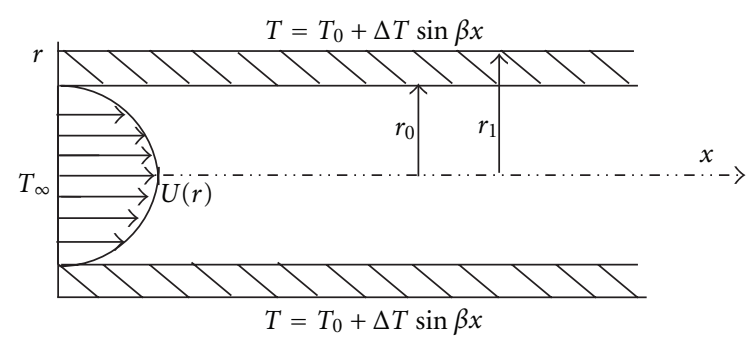

FIgURE 1: The axial section of channel.

\section{Mathematic Model}

In this section, the local energy balance equations, written separately for the solid region and for the fluid region, are solved analytically with the mentioned boundary condition.

Let us consider, an infinite circular duct with internal and external radii is, respectively, $r_{0}, r_{1}$. Since the channel has a symmetry with reference to the $r=0$, it is possible to study only half of the solution domain $\left(0 \leq r \leq r_{1}\right)$. A drawing of the section of the circular duct together with the boundary condition is shown in Figure 1.

With considering the fully developed convection of Newtonian fluid, constant thermophysical property, neglecting of axial heat conduction in both phase and viscose dissipation in the fluid phase, the local energy balance in fluid and solid phases is given by the following.

Fluid phase $\left(0<r<r_{0}\right)$ :

$$
\frac{\partial}{\partial r}\left(r \frac{\partial T}{\partial r}\right)-\frac{U(r)}{\alpha_{f}} r \frac{\partial T}{\partial x}=0
$$

Solid phase $\left(r_{0}<r<r_{1}\right)$ :

$$
\frac{1}{r} \frac{\partial}{\partial r}\left(r \frac{\partial T}{\partial r}\right)+\frac{\partial^{2} T}{\partial x^{2}}=0
$$

where $U(r)$ is the Poiseuille velocity distribution:

$$
U(r)=2 \bar{u}\left(1-\left(\frac{r}{r_{0}}\right)^{2}\right) .
$$

The equations must be solved together with following boundary conditions:

$$
\begin{aligned}
& \text { at } r=0 \Longrightarrow \frac{\partial T}{\partial r}=0, \\
& \text { at } r=r_{1} \Longrightarrow T=T_{0}+\Delta T \sin \beta x, \\
& \text { at } r=r_{0} \Longrightarrow T_{s}=T_{f}, \\
& \text { at } r=r_{0} \Longrightarrow k_{s} \frac{\partial T_{s}}{\partial r}=k_{f} \frac{\partial T_{f}}{\partial r} .
\end{aligned}
$$

With introducing the following dimensionless parameters:

$$
\eta=\frac{r}{r_{0}}, \quad \xi=\frac{x}{r_{0}}, \quad \theta=\frac{T-T_{0}}{\Delta T}, \quad \sigma=\frac{r_{1}}{r_{0}}, \quad \gamma=\frac{k_{s}}{k_{f}} \text {. }
$$

Equations (1), (2), and (4), respectively, transform to

$$
\begin{gathered}
\frac{\partial}{\partial \eta}\left(\eta \frac{\partial \theta}{\partial \eta}\right)-\operatorname{Pe}\left(1-\eta^{2}\right) \eta \frac{\partial \theta}{\partial \xi}=0, \quad 0<\eta<1, \\
\frac{1}{\eta} \frac{\partial}{\partial \eta}\left(\eta \frac{\partial \theta}{\partial \eta}\right)+\frac{\partial^{2} \theta}{\partial \xi^{2}}=0, \quad 1<\eta<\sigma,
\end{gathered}
$$

$$
\begin{aligned}
& \text { at } \eta=0 \Longrightarrow \frac{\partial \theta_{f}}{\partial \eta}=0 \\
& \text { at } \eta=\sigma \Longrightarrow \theta=\sin B \xi \\
& \text { at } \eta=1 \Longrightarrow \theta_{s}=\theta_{f}, \\
& \text { at } \eta=1 \Longrightarrow k_{s} \frac{\partial \theta_{s}}{\partial \eta}=k_{f} \frac{\partial \theta_{f}}{\partial \eta} \Longrightarrow \frac{\partial \theta_{f}}{\partial \eta}=\gamma \frac{\partial \theta_{s}}{\partial \eta}
\end{aligned}
$$

where $\mathrm{Pe}=2 r_{0}\left(\bar{u} / \alpha_{f}\right), B=\beta r_{0}$.

\section{Analytical Solution}

By assuming the axial position sufficiently far from the inlet section, the solution of (6) can be written as

$$
\theta(\xi, \eta)=\theta_{0}(\eta)+\theta_{1}(\eta) \sin (B \xi)+\theta_{2}(\eta) \cos (B \xi)
$$

By replacing (8) into (6), the $\theta_{0}(\eta), \theta_{1}(\eta)$, and $\theta_{2}(\eta)$ can be obtained from the following equations sets:

$$
\begin{aligned}
0<\eta<1 \Longrightarrow\left\{\begin{array}{l}
\frac{d}{d \eta}\left(\eta \frac{d \theta_{1}}{d \eta}\right)+\operatorname{Pe} B\left(1-\eta^{2}\right) \eta \theta_{2}=0 \\
\frac{d}{d \eta}\left(\eta \frac{d \theta_{2}}{d \eta}\right)-\operatorname{Pe} B\left(1-\eta^{2}\right) \eta \theta_{1}=0 \\
\frac{d}{d \eta}\left(\eta \frac{d \theta_{0}}{d \eta}\right)=0,
\end{array}\right. \\
1<\eta<\sigma \Longrightarrow\left\{\begin{array}{l}
\frac{1}{\eta} \frac{d}{d \eta}\left(\eta \frac{d \theta_{1}}{d \eta}\right)-B^{2} \theta_{1}=0 \\
\frac{1}{\eta} \frac{d}{d \eta}\left(\eta \frac{d \theta_{2}}{d \eta}\right)-B^{2} \theta_{2}=0 \\
\frac{1}{\eta} \frac{d}{d \eta}\left(\eta \frac{d \theta_{0}}{d \eta}\right)=0 .
\end{array}\right.
\end{aligned}
$$


And the boundary condition of equations sets (9) is given by

$$
\begin{aligned}
& \eta=0 \longrightarrow \frac{d \theta_{0}}{d \eta}=\frac{d \theta_{1}}{d \eta}=\frac{d \theta_{2}}{d \eta}=0, \\
& \eta=1 \Longrightarrow\left\{\begin{array}{l}
\frac{d \theta_{0, f}}{d \eta}=\gamma \frac{d \theta_{0, s}}{d \eta} \\
\frac{d \theta_{1, f}}{d \eta}=\gamma \frac{d \theta_{1, s}}{d \eta} \\
\frac{d \theta_{2, f}}{d \eta}=\gamma \frac{d \theta_{2, s}}{d \eta} \\
\theta_{0, f}=\theta_{0, s} \\
\theta_{1, f}=\theta_{1, s} \\
\theta_{2, f}=\theta_{2, s}, \\
\theta_{0}=0, \quad \theta_{1}=1, \quad \theta_{2}=0 .
\end{array}\right.
\end{aligned}
$$

In (9) it can be observed $\theta_{1}, \theta_{2}$ are coupled together, and $\theta_{0}$ is independent of $\theta_{1}, \theta_{2}$. The solution of boundary valued problem (9) with considering boundary conditions (10) yields $\theta_{0}=0$.

For uncoupling the $\theta_{1}, \theta_{2}$ equations, a new function $\psi$ is introduced as

$$
\psi=\theta_{1}+i \theta_{2}
$$

By employing (11), (9) can be collapsed into a boundary valued problem, such as (12)

$$
\begin{aligned}
& 0<\eta<1 \Longrightarrow \frac{1}{\eta} \frac{d}{d \eta}\left(\eta \frac{d \psi}{d \eta}\right)-i \operatorname{Pe} B\left(1-\eta^{2}\right) \psi=0, \\
& 1<\eta<\sigma \Longrightarrow \frac{1}{\eta} \frac{d}{d \eta}\left(\eta \frac{d \psi}{d \eta}\right)-B^{2} \psi=0 .
\end{aligned}
$$

Also boundary conditions (10) transform to

$$
\begin{aligned}
& \eta=\sigma \Longrightarrow \psi(\sigma)=1, \\
& \eta=0 \Longrightarrow \frac{d \psi}{d \eta}=0, \\
& \eta=1 \Longrightarrow \psi_{f}=\psi_{s}, \\
& \eta=1 \Longrightarrow \frac{d \psi_{f}}{d \eta}=\gamma \frac{d \psi_{s}}{d \eta} .
\end{aligned}
$$

By introducing the new parameters $\psi=\Phi / \eta, \omega=$ $\sqrt{-i \mathrm{Pe} B} / 2$, and $z=2 \omega \eta^{2}$ and replacing into (12), equation (15) is formed as

$$
\Phi^{\prime \prime}+\left(\frac{1}{4 z^{2}}+\frac{\omega}{2 z}-\frac{1}{4}\right) \Phi=0 .
$$

The solution of (15) which fulfills the condition $d \psi /\left.d \eta\right|_{\eta=0}=$ 0 is given by [13]

$$
\psi=\frac{C_{1}}{\eta} M_{\omega / 2,0}\left(2 \omega \eta^{2}\right),
$$

where $M$ is confluent hypergeometric function. $[14]$ :

$\psi$ function can be expressed as the following power series

$$
\begin{aligned}
\psi= & C 1 \frac{1}{\Gamma((1-\omega) / 2)}(2 \omega)^{1 / 2} \exp \left(-\omega \eta^{2}\right) \\
& \times \sum_{n=0}^{\infty} \frac{\Gamma(n+(1 / 2)-(\omega / 2))}{n ! n !}\left(2 \omega \eta^{2}\right)^{n}, \quad 0 \leq \eta \leq 1,
\end{aligned}
$$

where $\Gamma$ is the gamma function.

By employing the first- and second-type Bessel functions, respectively, $I, K$, the solution of (13) can be written as:

$$
\psi=C_{3} I(0, B \eta)+C_{4} K(0, B \eta), \quad 1 \leq \eta \leq \sigma .
$$

Constant $C_{1}, C_{3}$, and $C_{4}$ are obtained by using the mentioned boundary conditions in (14).

The Nusselt number can be obtained by employing (19)

$$
\begin{aligned}
\mathrm{Nu} & =\left.2 r_{0} \frac{\partial T}{\partial r}\right|_{f, r=r_{0}}\left[T\left(r_{0}, x\right)-T_{b}(x)\right]^{-1} \\
& =-\frac{2 \varphi(\xi)}{\theta(1, \xi)-\theta_{b}(\xi)},
\end{aligned}
$$

where $\varphi(\xi)$ is the dimensionless heat flux at solid-fluid interface:

$$
\begin{aligned}
\varphi(\xi) & =-\left.\frac{\partial \theta}{\partial \eta}\right|_{f, \eta=1} \\
& =-\left.\frac{d \theta_{1}}{d \eta}\right|_{f, \eta=1} \sin (B \xi)-\left.\frac{d \theta_{2}}{d \eta}\right|_{f, \eta=1} \cos (B \xi) \\
& =\varphi_{1} \sin (B \xi)+\varphi_{2} \cos (B \xi), \\
\varphi_{1} & =-\left.\frac{d \theta_{1}}{d \eta}\right|_{f, \eta=1}, \quad \varphi_{2}=-\left.\frac{d \theta_{2}}{d \eta}\right|_{f, \eta=1} .
\end{aligned}
$$

And $\theta_{b}(\xi)$ is the dimensionless bulk temperature:

$$
\theta_{b}(\xi)=4 \int_{0}^{1} \theta(\eta, \xi) \eta\left(1-\eta^{2}\right) d \eta
$$

By using the above equations, the Nusselt number is obtained as

$$
\mathrm{Nu}(\xi)=-\frac{2\left(\varphi_{1} \sin (B \xi)+\varphi_{2} \cos (B \xi)\right)}{\left(\theta_{1}(1)-\theta_{1 b}\right) \sin (B \xi)+\left(\theta_{2}(1)-\theta_{2 b}\right) \cos (B \xi)},
$$

where $\theta_{1 b}, \theta_{2 b}$ are, respectively, the real and imaginary part of following integral:

$$
\psi_{b}=4 \int_{0}^{1} \psi(\eta) \eta\left(1-\eta^{2}\right) d \eta
$$

One-time integration from (12) yields,

$$
\psi_{b}=\left.\frac{-4 i}{\operatorname{Pe} B} \frac{d \psi}{d \eta}\right|_{f, \eta=1}=\frac{4 i}{\operatorname{Pe} B}\left(\varphi_{1}+i \varphi_{2}\right) .
$$




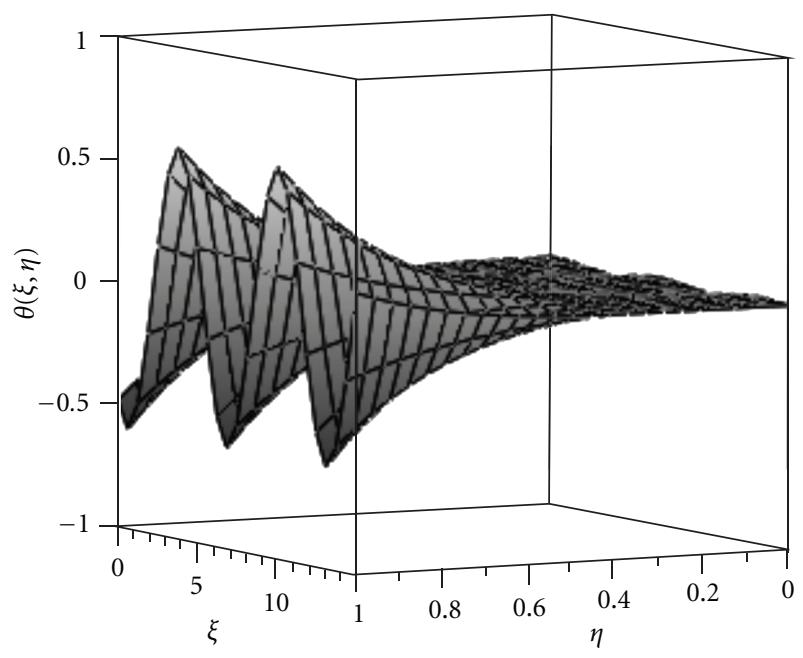

FIGURE 2: 3D plot of dimensionless temperature distribution versus $\xi$ and $\eta$.

On account of (24) the Nusselt number can be expressed as $\mathrm{Nu}(\xi)$

$$
=\frac{-2 B \operatorname{Pe}\left(\varphi_{1} \sin (B \xi)+\varphi_{2} \cos (B \xi)\right)}{\left(B \operatorname{Pe} \theta_{1}(1)+4 \varphi_{2}\right) \sin (B \xi)+\left(B \operatorname{Pe} \theta_{2}(1)-4 \varphi_{1}\right) \cos (B \xi)} .
$$

The average Nusselt number in an axial period is given by

$$
\overline{\mathrm{Nu}}=\frac{B}{2 \pi} \int_{0}^{2 \pi / B} N(\xi) d \xi
$$

Above integral has a singularity that is shown in (27):

$$
\xi_{n}=\tan ^{-1}\left(\frac{\left(\theta_{2}(1)-\left(\left(4 \varphi_{1}\right) /(B \mathrm{Pe})\right)\right)}{\left(\theta_{1}(1)+\left(\left(4 \varphi_{2}\right) /(B \mathrm{Pe})\right)\right)}\right)+\frac{n \pi}{B} .
$$

By employing the complex integral analysis and Residual theorem, the integral (26) is calculated as

$$
\overline{\mathrm{Nu}}=-\frac{8 \varphi_{1}\left[B \operatorname{Pe} \theta_{1}(1)+4 \varphi_{2}\right]+8 \varphi_{2}\left[B \operatorname{Pe} \theta_{2}(1)-4 \varphi_{1}\right]}{\left(B \operatorname{Pe} \theta_{1}(1)+4 \varphi_{2}\right)^{2}+\left(B \operatorname{Pe} \theta_{2}(1)-4 \varphi_{1}\right)^{2}} .
$$

The simplified form of (28) is given by

$$
\overline{\mathrm{Nu}}=-\frac{8 \varphi_{1} B \operatorname{Pe} \theta_{1}(1)+8 \varphi_{2} B \operatorname{Pe} \theta_{2}(2)}{\left(B \operatorname{Pe} \theta_{1}(1)+4 \varphi_{2}\right)^{2}+\left(B \operatorname{Pe} \theta_{2}(1)-4 \varphi_{1}\right)^{2}} .
$$

And finally $\overline{\mathrm{Nu}}$ can be rewritten as

$$
\overline{\mathrm{Nu}}=\left.\frac{4 B \operatorname{Pe}}{\left|B \operatorname{Pe} \psi(1)-4 i\left(\varphi_{1}+\varphi_{2}\right)\right|^{2}} \frac{d|\psi|^{2}}{d \eta}\right|_{f, \eta=1} .
$$

\section{Result and discussion}

In Figure 2 three-dimensional plot of dimensionless temperature distribution versus $\xi$ and $\eta$ is shown, for

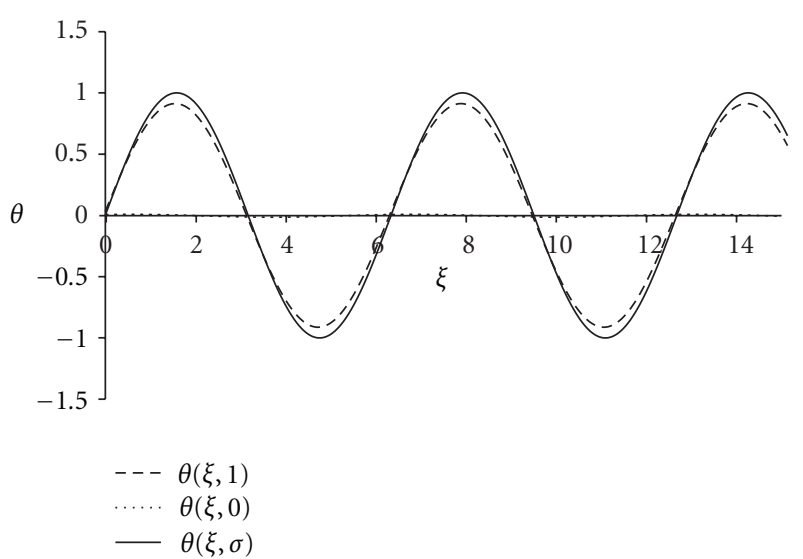

FIGURE 3: Dimensionless temperature distribution at $\eta=0,1, \sigma$ versus $\xi$.

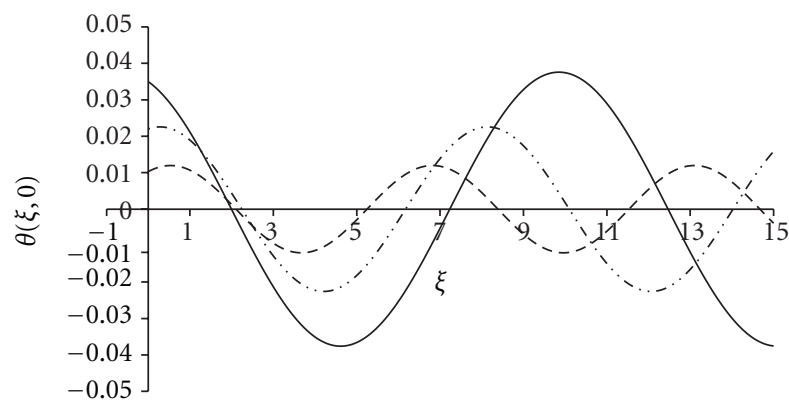

$$
\begin{aligned}
--B & =1 \\
-\cdots & B=0.8 \\
-B & =0.6
\end{aligned}
$$

FIgure 4: Dimensionless temperature distribution at $\eta=0$ for $B=$ $0.3,0.6$, and 0.8 versus $\xi$.

$\sigma=1.15, \gamma=1.5, \mathrm{Pe}=100$, and $B=1$. Figure 2 shows by moving from wall to the center of duct the oscillation amplitude of temperature decreases, but in the center of duct the oscillation amplitude is not zero and Figure 3 confirms it. In this figure the dimensionless temperature versus $\xi$, for $\sigma=1.15, \gamma=1.5, \mathrm{Pe}=100$, and $B=0.1$ that is displayed. In Figure 3 it can be observed by closing to the center of duct not only the oscillation amplitude decreases, but also the sensing of wall temperature alteration is done with more longitudinal delay. In Figures 4 and 5 The dimensionless temperature distribution at the center of duct is reported for $\sigma=1.15, \gamma=1.5$, and $\mathrm{Pe}=100$ and various values of $B, \mathrm{Pe}$, respectively. The Figure 4 shows that increasing in $B$ causes a decrease in temperature oscillation amplitude in flow field.

The reason of this phenomenon is related to the effect of conduction accompanied by convection heat transfer. Because of conduction heat transfer in $\mathrm{Y}$ direction, each point of fluid senses wall temperature alteration, immediately and without any longitudinal delay. That means by only taking into account conduction, when the wall temperature is Max at an axial position, for example $\mathrm{X}_{0}$, the temperature of all points in fluid with same axial position as $\mathrm{X}_{0}$, is Max, 


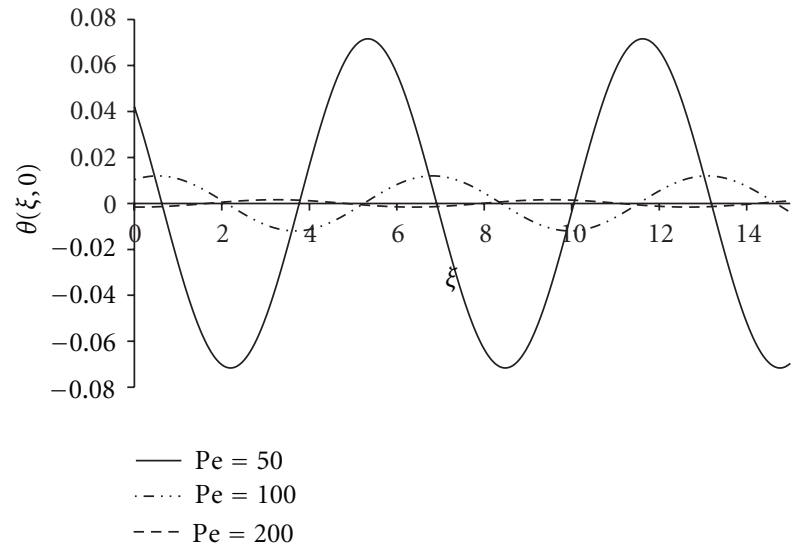

Figure 5: Dimensionless temperature distribution at $\eta=0$ for $\mathrm{Pe}=$ $20,50,100$, and 200 versus $\xi$.

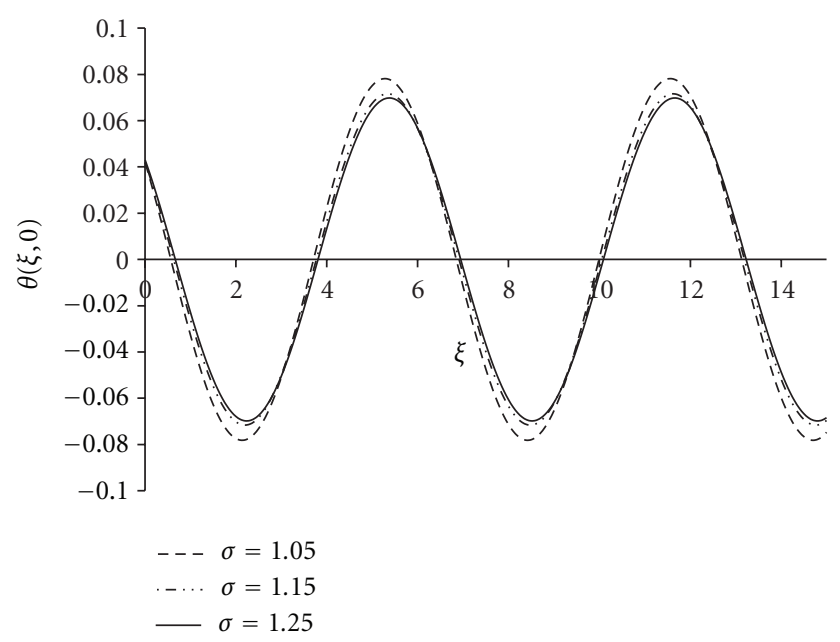

Figure 6: Dimensionless temperature distribution at solid-fluid interface for $\sigma=1.05,1.15,1.25$ versus $\xi$.

but with minus gradient in $\mathrm{Y}$ direction. About convection heat transfer, it should be mentioned that convection phenomenon transfers the upstream pointes property to downstream pointes in $\mathrm{X}$ direction; as a result of only taking into account convection, when the wall temperature is Max at an axial position, as $\mathrm{X}_{0}$, the points with $\mathrm{X}_{0}$ axial position are affected by upstream point only that has lower temperature in comparison to Max temperature. By increasing in $B$, frequency of oscillation increases, and it causes more difference between Max temperature; and upstream point temperature, as a result, contrast between convection and conduction increases and amplitude decreases.

In Figure 5 it can be observed that Pe number and $B$ have a same effect on oscillation amplitude of dimensionless temperature. According to Figure 5 an increase in Pe number causes an amplitude reduction. The reason of this behavior is also related to the difference between conducted and conduction phenomenon. Since Pe number is convection to conduction heat transfer rate ratio, increase in Pe number
TABLE 1: The values of the mean Nusselt number for $\sigma=1.15, \gamma=$ 1.5 .

\begin{tabular}{lccc}
\hline B.Pe & $\mathrm{Pe}=10$ & $\mathrm{Pe}=50$ & $\mathrm{Pe}=100$ \\
\hline 10 & 3.556 & 3.951 & 4.404 \\
20 & 4.061 & 4.210 & 4.581 \\
50 & 5.425 & 5.817 & 5.753 \\
100 & 8.881 & 6.115 & 6.010 \\
150 & 11.982 & 6.843 & 6.565 \\
200 & 16.255 & 8.005 & 7.684 \\
300 & 23.412 & 8.569 & 7.951 \\
\hline
\end{tabular}

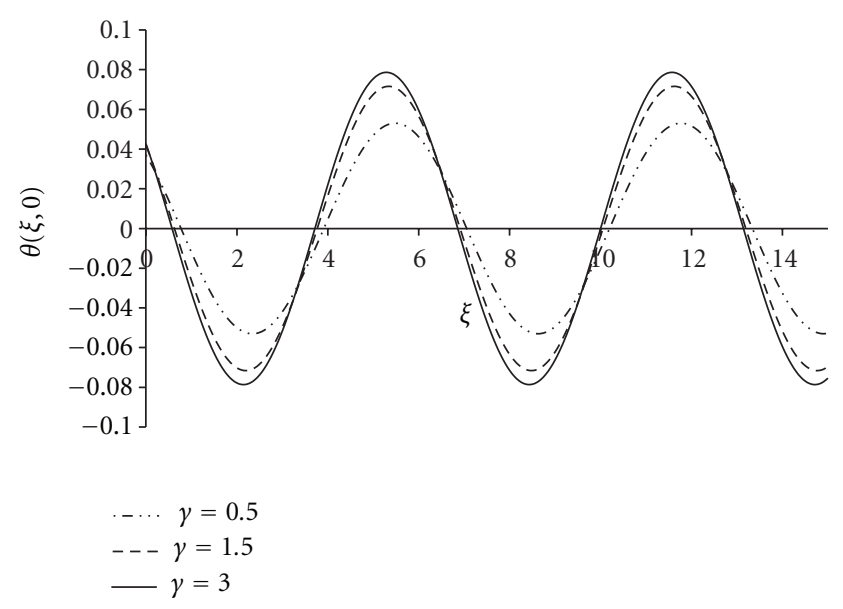

FIgURE 7: Dimensionless temperature distribution at solid-fluid interface for $\gamma=0.5,1.5,3.0$ versus $\xi$.

leads to increase in convection against conduction heat transfer as a result the temperature distribution in the fluid phase is more affected by upstream condition in comparison to wall boundary condition. In Figure 6 the dimensionless temperature of solid and fluid phases interface against $\xi$ for different values of $\sigma$ is displayed.

Figure 6 shows since increasing in wall thickness is followed with a decrease in conduction heat transfer, the oscillation amplitude in flow field decreases.

The longitudinal dimensionless temperature distribution is observed in Figure 7 for several values of $\gamma$. In the Figure 7 it is seen that increase in heat conductivity of solid phase is followed with increase in oscillation amplitude, because of lower temperature gradient.

In Table 1, the values of the mean Nusselt number, evaluated through (30), are reported versus B.Pe and Pe. The table shows that the mean Nusselt number is an increasing monotonic function of $B$. Since the temperature gradient in $\mathrm{Y}$ direction has a direct relation with $B$, as a result larger value of $B$ leads to large value of Nusselt number.

\section{Conclusion}

In the present paper, the heat transfer problem has been studied for the laminar forced convection of a fluid in a circular duct. The duct walls have finite width, and the 
temperature on the external boundaries varies longitudinally with sinusoidal law. The local energy balance equation has been solved analytically, with reference to the thermally developed region. Under this assumption, the temperature distribution is a periodic function of the axial direction, and the period is equal to the period of the wall temperature distribution and the following result is obtained.

(i) The oscillation amplitude of the temperature field decreases by increasing the dimensionless frequency $B$ and Peclet number.

(ii) Increasing in The dimensionless frequency $B$ is followed with increase an average Nusselt number.

\section{Nomenclatures}

$\begin{array}{ll}B: & \text { Dimensionless frequency } \\ C 1, C 2, C 3, C 4: & \text { Constants coefficient } \\ i: & \text { Complex unit } \\ k: & \text { Thermal conductivity } \\ n: & \text { Positive integer } \\ M: & \text { Confluent hypergeometricfunction } \\ \mathrm{Nu}: & \text { Local Nusselt number } \\ \overline{\mathrm{Nu}}: & \text { Mean Nusselt number } \\ r: & \text { Radial coordinate } \\ r_{0}: & \text { Internal radios } \\ r_{1}: & \text { External radios } \\ T: & \text { Temperature } \\ u: & \text { Fluid velocity } \\ \bar{u}: & \text { Mean fluid velocity } \\ x: & \text { Axial coordinate. }\end{array}$

\section{Greek Symbols}

$\begin{array}{ll}\alpha: & \text { Thermal diffusivity } \\ \beta: & \text { Frequency } \\ \gamma: & \text { Dimensionless parameter, defined in (5) } \\ \Delta T: & \text { Oscillation temperature of external wall } \\ \xi: & \text { Dimensionless axial coordinate } \\ \eta: & \text { Dimensionless radial coordinate } \\ \theta: & \text { Dimensionless temperature } \\ \theta_{0}, \theta_{1}, \theta_{2}: & \text { Dimensionless function, defined in }(8) \\ \Phi: & \text { Dimensionless function, defined in }(15) \\ \varphi_{1}, \varphi_{2}: & \text { Dimensionless function, defined in }(21) \\ \sigma: & \text { Dimension radios of duct } \\ \psi: & \text { Dimensionless complex function, defined in } \\ & \text { (11). }\end{array}$

\section{Subscripts}

$b$ : Bulk quantity

$f$ : Fluid

s: Solid.

\section{References}

[1] A. Barletta and E. Zanchini, "Laminar forced convection with sinusoidal wall heat flux distribution: axially periodic regime," Heat and Mass Transfer, vol. 31, no. 1-2, pp. 41-48, 1995.

[2] A. Barletta, E. R. di Schio, G. Comini, and P. D’Agaro, "Conjugate forced convection heat transfer in a plane channel: longitudinally periodic regime," International Journal of Thermal Sciences, vol. 47, no. 1, pp. 43-51, 2008.

[3] A. Barletta and E. Rossi di Schio, "Effects of viscous dissipation on laminar forced convection with axially periodic wall heat flux," Warme-und Stoffubertragung Zeitschrift, vol. 35, no. 1, pp. 9-16, 1999.

[4] A. Barletta and E. Rossi di Schio, "Periodic forced convection with axial heat conduction in a circular duct," International Journal of Heat and Mass Transfer, vol. 43, no. 16, pp. 29492960, 2000.

[5] A. Barletta and E. Rossi di Schio, "Forced convection in a circular duct with periodic boundary conditions and axial heat conduction," Heat and Technology, vol. 18, no. 1, pp. 93-101, 2000.

[6] A. Barletta, E. Rossi di Schio, G. Comini, and P. D'Agaro, "Wall coupling effect in channel forced convection with streamwise periodic boundary heat flux variation," International Journal of Thermal Sciences, vol. 48, no. 4, pp. 699-707, 2009.

[7] G. Comini, S. del Giudice, and C. Nonino, Finite Element Analysis in Heat Transfer, Taylor \& Francis, Washington, DC, USA, 1994.

[8] G. Comini, G. Cortella, and M. Manzan, "Streamfunctionvorticity-based finite-element formulation for laminarconvection problems," Numerical Heat Transfer B, vol. 28, no. 1, pp. 1-22, 1995.

[9] C. Nonino and G. Comini, "An equal-order velocity-pressure algorithm for incompressible thermal flows, part 1: formulation," Numerical Heat Transfer B, vol. 32, no. 1, pp. 1-15, 1997.

[10] C. Nonino and G. Comini, "Finite-element analysis of convection problems in spatially periodic domains," Numerical Heat Transfer B, vol. 34, no. 4, pp. 361-378, 1998.

[11] G. Comini, C. Nonino, and S. Savino, "Convective heat and mass transfer under dehumidifying conditions," in Progress in Computational Heat and Mass Transfer, R. Bennacer, Ed., pp. 711-722, Editions TEC and COC-Lavoisier, Paris, France, 2005.

[12] A. Barletta, E. Rossi di Schio, and L. Selmi, "Thermal nonequilibrium and viscous dissipation in the thermal entrance region of a Darcy flow with streamwise periodic boundary conditions," Journal of Heat Transfer, vol. 133, no. 7, Article ID 072602, 2011.

[13] M. Abramowitz and I. A. Stegun, Handbook of Mathematical Function, Dover, New York, NY, USA, 1970.

[14] H. A. Lauwerier, "The use of confluent hypergeometric functions in mathematical physics and the solution of an eigenvalue problem," Applied Scientific Research, vol. 2, no. 1, pp. 184-204, 1951. 

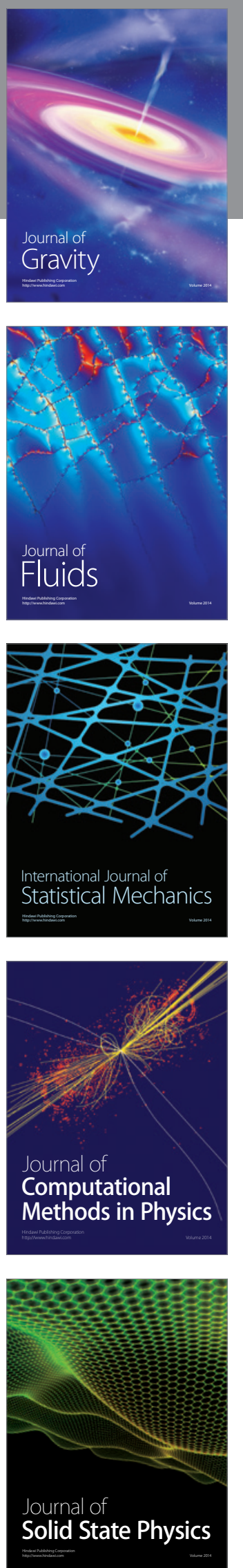

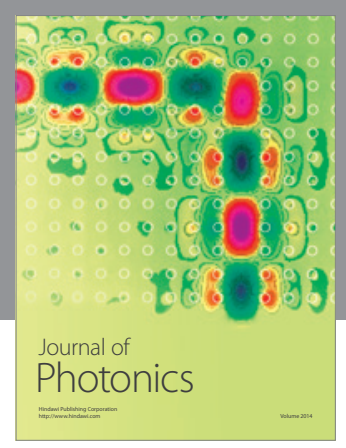

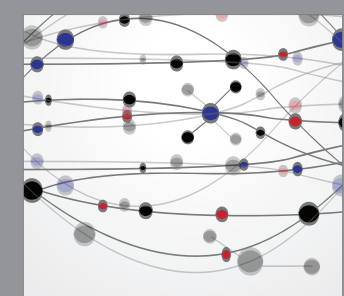

The Scientific World Journal
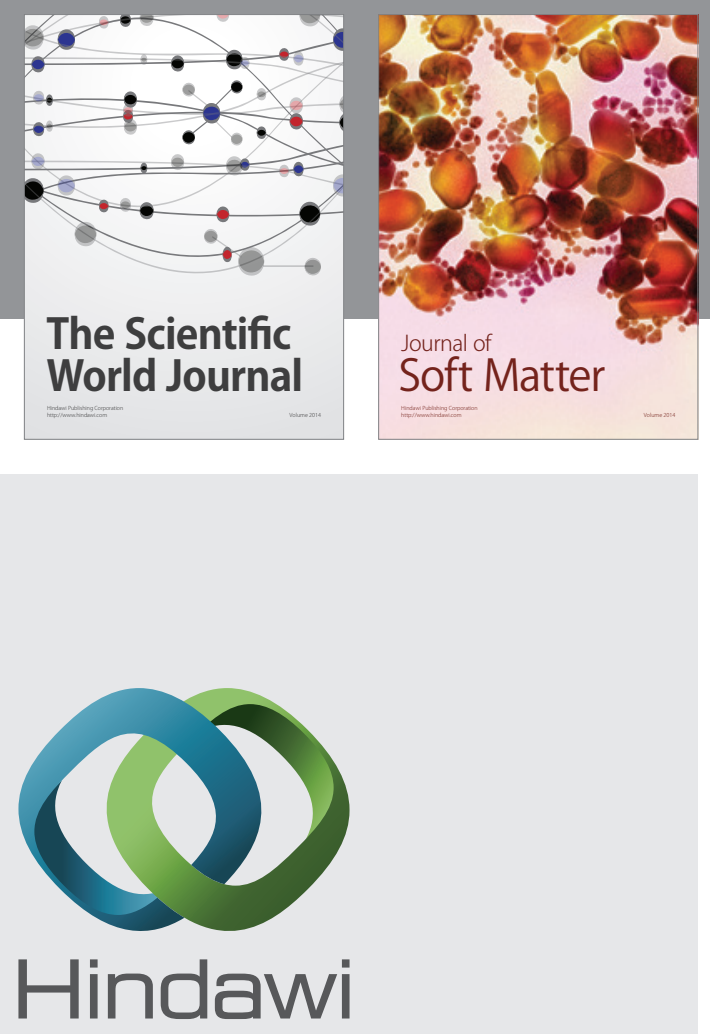

Submit your manuscripts at

http://www.hindawi.com
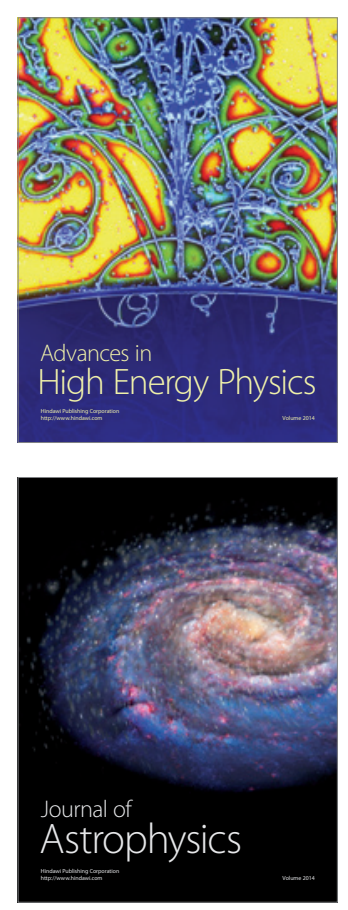
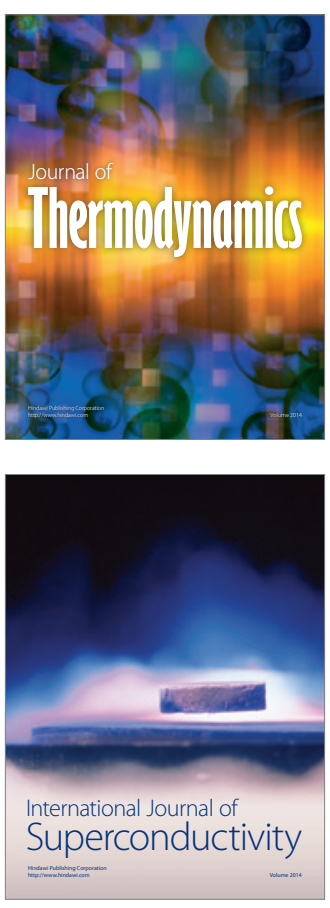
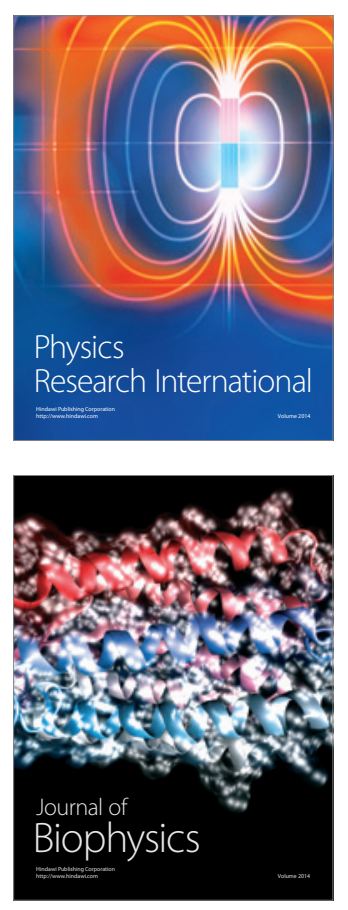
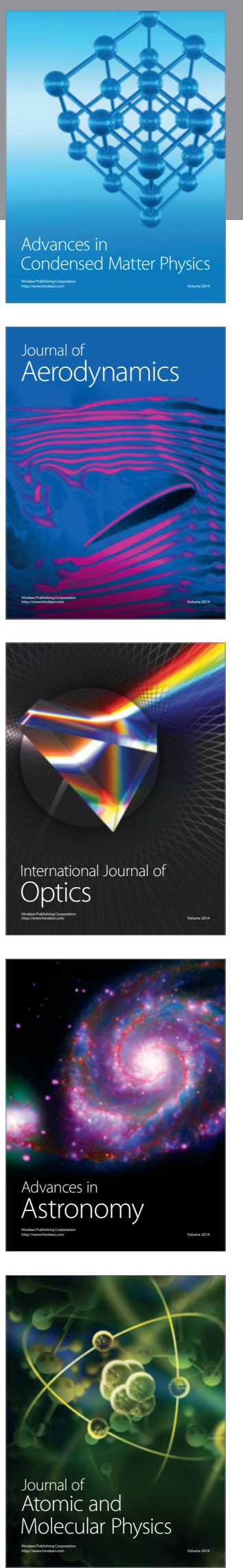